Background and aims Disturbed cerebral oxygenation during the neonatal period might pose preterm infants at risk for neurological deficits. Our aim was to determine whether regional cerebral tissue oxygen saturation $\left(\mathrm{r}_{\mathrm{c}} \mathrm{SO}_{2}\right)$ and fractional tissue oxygen extraction (FTOE), measured by near-infrared spectroscopy, were associated with neurodevelopmental outcome of preterm infants at 2-3 years of age.

Methods We included 83 preterm infants (gestational age $<32$ weeks) and measured $\mathrm{r}_{\mathrm{c}} \mathrm{SO}_{2}$, and calculated FTOE on days 1, 2, 3, 4, 5,8 , and 15 after birth: $\left(\mathrm{tcSaO}_{2}-\mathrm{r}_{\mathrm{c}} \mathrm{SO}_{2}\right) / \mathrm{tcSaO}$. Additionally, we determined the area under the curve (AUC) of $\mathrm{r}_{\mathrm{c}} \mathrm{SO}_{2}$ and FTOE during the first 2 weeks. Cognitive, motor, neurological and behavioural outcome was determined at 2-3 years of age. Multiple linear regression analyses were used to determine whether $\mathrm{r}_{\mathrm{c}} \mathrm{SO}_{2}$ and FTOE contributed to outcome.

Results We included 67 infants for follow-up. Lower quartile $\left(\mathrm{P}_{25-50}\right)$ and highest quartile $\left(\mathrm{P}_{75-100}\right)$ of $\mathrm{r}_{\mathrm{c}} \mathrm{SO}_{2}$ values on day 1 were associated with poorer cognitive outcome ( $\mathrm{p}=0.044$ and $\mathrm{p}=0.008$, respectively). Lower AUC of $\mathrm{r}_{\mathrm{c}} \mathrm{SO}_{2}$ was associated with poorer cognitive outcome $(\mathrm{p}=0.014)$. Lower quartile $\left(\mathrm{P}_{25-50}\right) \mathrm{AUC}$ of $\mathrm{r}_{\mathrm{c}} \mathrm{SO}_{2}$ was associated with poorer fine motor outcome ( $p=0.004)$. The amount of time $\mathrm{r}_{\mathrm{c}} \mathrm{SO}_{2}<50 \%$ on day 1 was negatively associated with gross motor outcome $(\mathrm{p}=0.002)$. The highest quartile of FTOE values on day 1 was associated with poorer total motor outcome $(\mathrm{p}=0.041)$.

Conclusions Neurodevelopmental outcome at 2-3 years of age was associated with cerebral oxygen saturation during the first 2 weeks after birth in preterm infants. Both high and low $\mathrm{r}_{\mathrm{c}} \mathrm{SO}_{2}$ values had a negative influence on neurodevelopmental outcome.

\section{GUIDED IMAGERY: BEYOND PROCEDURAL FEAR AND PAIN - TECHNIQUES FOR CHILDREN, ADOLESCENTS AND YOU, THE HEALTH PROFESSIONAL}

doi:10.1136/archdischild-2012-302724.0110

B Whitaker. Top-Down Pain Control, Redhill, UK

'You have had blood tests before, haven't you?'

"Yeah, but never this exciting!"

The response to the question quoted above came from an 11 year-old boy with special needs following his venepuncture in imagery'. The procedure was 'exciting' because he was playing his favourite game - cricket. He hit a fantastic score, got 'run out' and won the game, all in the space of seven (7) minutes from the beginning to the end of the procedure.

Guided Imagery is a therapeutic technique that enables a topdown shift in experience from pain and fear to a chosen reality in imagery. It is used to help a child gain a sense of being in control in the face of fear, anxiety and pain during medical procedures.

This presentation will include:

- an overview of guided imagery

- video of a child undergoing a procedure in imagery;

- mindfulness and Acceptance and Commitment Therapy ACT (Hayes \& Smith, 2005) in relation to procedural pain and fear; and,

- moving from patterns of 'avoidance and bravery' to 'acceptance and achievement' in managing pain.

Delegates attending this presentation will have an opportunity to engage in a mindfulness exercise.

Hayes, S.C. \& Smith, S. (2005). Get out of your mind and into your life: The new acceptance and commitment therapy. Oakland: New Harbinger.
A Memarizadeh, N Beheshtipour, F Hashemi, S Porarian. Shiraz University of Medical Sciences, Shiraz, Iran

Objective Pain is a multi-dimensional and complex experience which influences all people physically, emotionally and socially. The process of care and pain relief is the duty of nurses. Today, pain is assessing as the fifth human vital signs. Therefore, the standards of nursing care should be prioritized. The objective of this study was to assess the efficacy of kangaroo care (KC) on pain severity during the heel stick in premature infants.

Material and method In this interventional study, a total of 20 premature infants recruited during 4 months were enrolled in this study. The inclusion criteria were premature infants with gestational age 27-36 weeks and aged 3-28 days, stable respiratory status. In need of heel stick and Apgar score more than 6 at 5 minutes. In this randomized interventional study the blood is taken from each infant once by using $\mathrm{KC}$ and another time by incubator care(IC). In each method, PIPP was used to evaluate the severity of pain. Data were analyzed using by SPSS for Windows. Paired sample t-test was used for data analysis.

Findings The severity of pain as the result of heel stick is different in 2 methods. The mean pain score in the $\mathrm{KC}$ method was $4.9 \pm 2.92$ vs IC $11 \pm 4.22$ and that is, significantly lower than the (IC) $(P<0.001)$.

Conclusion $\mathrm{KC}$ that starting 30 minutes before and continuing 10 minutes after heel stick was found to be effective in decreasing pain before and after heel stick in premature infants.

\section{A PILOT STUDY INVESTIGATING THE USE OF HEATED HUMIDIFIED OXYGEN AND DRY OXYGEN THERAPY IN CHILDREN WITH ACUTE RESPIRATORY ILLNESS}

doi:10.1136/archdischild-2012-302724.0112

LC Whelan, E McCall. Paediatric Intensive Care Unit, Starship Children's Health, Auckland, New Zealand

Background and aims Low flow oxygen can be delivered either as a dry or heated humidified gas. Little evidence exists about which is most beneficial to the paediatric patient. The aim of the study is to investigate whether children aged $<2$ years with acute respiratory illness requiring low flow oxygen ( $<2$ litres) would benefit from heated humidified oxygen when compared to dry oxygen therapy.

Methods A prospective randomised controlled pilot study of 117 children aged $<2$ years with an acute respiratory illness requiring low flow oxygen therapy. 54 participants were randomised to receive dry oxygen and 63 participants were randomised to receive heated humidified oxygen. Hours on oxygen therapy, hours to ready to discharge and hours to hospital discharge were recorded.

Results Median hours on dry oxygen therapy totalled 43 hours versus 39 hours in the heated humidified oxygen group. Wilcoxon rank test $(p=0.05$ ) returned $P$ value 0.77 . Hours from randomisation to ready to discharge equated to 66 hours dry oxygen and 52 hours heated humidified oxygen. Wilcoxon rank test $(\mathrm{p}=0.05)$ calculated $p=0.36$. The total median length of hospital stay was 53 hours in the dry oxygen group and 47 hours in the heated humidified oxygen group. Wilcoxon rank test $(\mathrm{p}=0.05)$ determined $\mathrm{p}=0.70$.

Conclusion No statistically significant difference in hours on oxygen therapy, time to ready to discharge and length of hospital stay between the dry oxygen and heated humidified group. No significant benefit to the administration of heated humidified oxygen at low flows was found. 